\title{
Severe Drug-induced Liver Injury Associated with Prolonged Use of Linezolid
}

\author{
Liesbet De Bus • Pieter Depuydt • Louis Libbrecht - Linos Vandekerckhove • \\ Joke Nollet • Dominique Benoit • Dirk Vogelaers • Hans Van Vlierberghe
}

Published online: 1 April 2010

(C) American College of Medical Toxicology 2010

\begin{abstract}
This study aims to describe a patient developing concomitant severe liver failure and lactic acidosis after long-term treatment with linezolid. A 55-year-old Caucasian woman developed concomitant severe liver failure and lactic acidosis after a treatment with linezolid for 50 days because of infected hip prosthesis. Other causes of liver failure and lactic acidosis were excluded by extensive diagnostic workup. A liver biopsy showed microvesicular steatosis. As linezolid toxicity was considered to be the cause of the lactic acidosis and the severe hepatic failure, the antibiotic was withdrawn. After 4 days of supportive therapy and hemodialysis, the serum lactate level returned
\end{abstract}

The patient and relatives provided written informed consent for the publication of this case report.

L. De Bus $(\bowtie) \cdot$ P. Depuydt $\cdot$ J. Nollet $\cdot$ D. Benoit

Department of Intensive Care, University Hospital,

12K12IB, 185 De Pintelaan,

9000 Ghent, Belgium

e-mail: liesbetdebus@hotmail.com

\section{P. Depuydt}

Heymans Institute of Pharmacology, University Hospital,

Ghent, Belgium

L. Libbrecht

Department of Anatomopathology, University Hospital,

Ghent, Belgium

L. Vandekerckhove $\cdot$ D. Vogelaers

Department of Infectious Diseases, University Hospital,

Ghent, Belgium

H. Van Vlierberghe

Department of Hepatology, University Hospital,

Ghent, Belgium within normal limits. The prothrombin time ratio and thrombocytes recovered within 2 weeks. Bilirubin levels normalized within 14 weeks. Since no other cause could be identified, liver injury was considered to be drug-related. Resolution of the hepatotoxicity occurred after discontinuation of linezolid, supportive treatment measures, and hemodialysis. Both lactic acidosis and microvesicular steatosis after the use of linezolid are related to mitochondrial dysfunction. The Council for International Organizations of Medical Sciences/Roussel Ucalf Causality Assessment Method scale revealed that the adverse drug event was probable. Prolonged exposure to linezolid may induce severe hepatotoxicity. Clinicians should be aware of this possible adverse effect especially in case of long-term treatment.

Keywords Linezolid - Liver failure .

Microvesicular steatosis $\cdot$ Lactic acidosis

\section{Introduction}

Infection of prosthetic devices is frequently caused by Gram-positive pathogens and requires prolonged antibiotic treatment in order to achieve microbial eradication. When methicillin-resistant staphylococci are involved, linezolid is a recommendable therapeutic option as it shows excellent activity against these pathogens and as it has a $100 \%$ oral bioavailability [1]. As such, it can be administered orally and on an outpatient basis, whereas treatment with glycopeptides requires intravenous access, which often implies prolonged hospitalization.

Serious side effects of linezolid therapy have been described, including bone marrow toxicity, peripheral and optic neuropathy, and lactic acidosis [2-4]. Lactic acidosis 
has been reported almost exclusively in patients treated for longer than the maximum recommended duration of 28 days. In this report, we describe a case of severe druginduced liver injury (DILI) related to the use of linezolid.

\section{Case Report}

A 55-year-old woman with rheumatoid arthritis was hospitalized for malnutrition and chronic infection in a prosthetic hip. Upon admission, alkaline phosphatase and gamma-glutamyltransferase levels were elevated, respectively, four and six times the upper limit values (Table 1). The hip prosthesis was removed and oxacillin was started. After 2 weeks, her labs worsened (Table 1), so this was discontinued and glycopeptides (initially vancomycin which was switched to teicoplanin) were started. The transient elevation in transaminases, alkaline phosphatase, and gamma-glutamyltransferase (without increase in bilirubin) prompted a diagnostic workup for liver disease. On abdominal ultrasound, biliary ducts were not dilated, liver parenchyma had a homogeneous aspect, and portal and hepatic circulations were normal. Hepatitis viral serology was negative. Ceruloplasmin, serum copper concentration, and alpha-1-antitrypsin were normal. Transaminase levels returned to normal limits within 1 week, followed by a slower decrease of alkaline phosphatase and gammaglutamyltransferase levels, which returned to the patients' baseline values (Table 1).

After 5 weeks on glycopeptides, she developed renal toxicity (on vancomycin) and thrombocytopenia (on teicoplanin), so these were discontinued and linezolid (600 mg every $12 \mathrm{~h}$ ) was initiated. Liver function tests at the start of linezolid (day 0) are detailed in Table 1. Meropenem (1 g every $8 \mathrm{~h}$ ) was added for coverage of Gram-negative pathogens. With this antibiotic regimen, the patient improved clinically, and inflammatory parameters decreased gradually, but symptoms of anorexia and weight loss persisted, necessitating placement on parenteral nutrition.

She was discharged from the hospital 4 months after the removal of the prosthesis. Six days later, she was readmitted to the emergency department with general weakness and itching, right hypochondrial and epigastric pain, nausea, and vomiting for 4 days. Her family had noticed yellow discoloration of the sclerae for 2 days. At that time, she was taking linezolid and meropenem for 50 days and was receiving home total parenteral nutrition (Structokabiven $12 \mathrm{gN}$ (Fresenius ${ }^{\circledR}$ ), supplemented with vitamins and oligo-elements) for 36 consecutive days. Additional medication consisted of vitamin $\mathrm{D}$, calcium, domperidone, esomeprazole, enoxaparin, and dorzolamide-timolol eye drops. Upon admission, she had a temperature of $37^{\circ} \mathrm{C}$, a pulse rate of $103 / \mathrm{min}$, a blood pressure of $110 / 60 \mathrm{mmHg}$, and a respiratory rate of 36 breaths per minute. Clinical examination showed an icteric and dehydrated patient in poor general condition. She was admitted to the intensive care unit (ICU) because of progressive encephalopathy, grade 3 (presence of apathy, somnolence, and disorientation). The abdomen was tender, especially in the right hypochondriac region, but there was no peritoneal irritation. On admission, blood analysis showed a severe lactic acidosis: pH7.27 (normal 7.35-7.45), bicarbonate $11.2 \mathrm{mmol} / \mathrm{l}$ (normal 22-26), lactate $121 \mathrm{mg} / \mathrm{dl}$ (normal 916), and cholestasis (gamma-glutamyltransferase $559 \mathrm{U} /$ 1 (normal 9-36), alkaline phosphatase 2,486 U/1 (normal 30120 ), total bilirubin $12.1 \mathrm{mg} / \mathrm{dl}$ (normal $0.3-1.2$ ), direct bilirubin $8.83 \mathrm{mg} / \mathrm{dl}$ (normal 0-0.30)). Transaminases were slightly elevated: aspartate aminotransferase $97 \mathrm{U} / 1$ (normal 0-31) and alanine aminotransferase $113 \mathrm{U} / 1$ (normal 7-31); and thrombocytopenia was present: $84,000 / \mu 1$ (normal 177,000-393,000). The prothrombin time ratio was $50 \%$

Table 1 Time course of laboratory test results parameter (reference range)

\begin{tabular}{|c|c|c|c|c|c|c|c|}
\hline & ALT (7-31 U/1) & AST (0-31 U/1) & ALP (30-120 U/1) & GGT (9-36 U/1) & TotBili $(0.3-1.2 \mathrm{mg} / \mathrm{dl})$ & PTI (70-120\%) & Lactate $(9-16 \mathrm{mg} / \mathrm{dl})$ \\
\hline$D-64$ & 49 & 42 & 453 & 181 & 1.0 & 90 & - \\
\hline$D-42$ & 33 & 45.1 & 525 & 178.1 & 0.53 & 108 & - \\
\hline $\mathrm{D}-34$ & 547 & 755 & 819 & 250.1 & 0.41 & 75 & - \\
\hline$D-6$ & 32 & 43.6 & 300.4 & 102.6 & 0.35 & 124 & - \\
\hline $\mathrm{D} 0^{\mathrm{a}}$ & 44 & 48.8 & 226.5 & 71.1 & 0.31 & 71 & 8.1 \\
\hline $\mathrm{D}+34$ & 10 & 15 & 830 & 497 & 0 & 86 & - \\
\hline $\mathrm{D}+50$ & 102 & 89.8 & 2,275 & 486.3 & 11.37 & 50 & 113.4 \\
\hline $\mathrm{D}+54$ & 223 & 227 & 1,515 & 297 & 12.86 & 56 & 13.4 \\
\hline $\begin{array}{l}D+ \\
104\end{array}$ & 15 & 9.5 & 432.9 & 130.3 & 0.99 & 100 & - \\
\hline
\end{tabular}

$A L T$ alanine aminotransferase, $A S T$ aspartate aminotransferase, $A L P$ alkaline phosphatase, TotBili total bilirubin, GGT gamma-glutamyltransferase, $P T I$ prothrombin time index, $D$ day

${ }^{\mathrm{a}} \mathrm{D} 0$ refers to the day linezolid was started 
(normal 70-120\%). In European laboratories, the prothrombin time is usually expressed as a ratio (rather than as seconds) (patient's prothrombin time divided by mean normal prothrombin time (pooled plasma)). The serum ammonia was elevated up to $86 \mu \mathrm{mol} / 1$ (normal 11-48). She also had a mild elevation of the serum creatinine on admission of $1.07 \mathrm{mg} / \mathrm{dl}$ (normal 0.55-0.96). In the presence of impaired synthetic function (decreased prothrombin time and spontaneous hypoglycemia), encephalopathy and icterus having occurred within less than 6 weeks, a diagnosis of acute liver failure was made. Initially, she was hemodynamically and respiratory stable. There were no signs of cardiac ischemia based on cardiac enzymes, electrocardiography, and transthoracic echocardiography. There were no focal signs of intercurrent infection. Linezolid, meropenem, and total parenteral nutrition were discontinued, and continuous hemodialysis was started because of persistent lactic acidosis within $24 \mathrm{~h}$ of ICU admission.

Further investigations were performed. Viral serology and antimitochondrial antibodies were negative. Antinuclear factor was positive, which was attributed to rheumatoid arthritis. Anti-smooth-muscle antibodies were mildly elevated. There was no hepatomegaly or dilatation of the biliary ducts on abdominal ultrasound and magnetic resonance choledochopancreaticography. The liver parenchyma had a heterogenous aspect on magnetic resonance imaging. The duplex Doppler examination of the liver and portal system was normal. A computed tomography scan of the abdomen showed a diffuse edematous thickening of the small intestine wall and colon wall and the presence of intra-abdominal free fluid. During a diagnostic explorative laparoscopy, for which she was electively intubated on day 2 of ICU admission, there were no arguments for ischemia of the small and large intestine. The blood supply to all other abdominal organs was preserved. A liver with a rough surface but normal size was seen. A subcapsular liver biopsy was performed. Light microscopy revealed preserved architecture of the liver parenchyma, with the presence of diffuse and mainly microvesicular steatosis. The pas-diastase staining revealed several ceroid macrophages, which were mainly localized in the centrilobular area. Some interlobular bile ducts showed a flattened, damaged epithelium, and the staining for cytokeratin 7 revealed that some portal tracts contained no bile duct. In agreement with these findings, the cytokeratin 7 staining also showed periportal ductular reaction and cholate stasis. Overall, this subcapsular liver biopsy showed diffuse microvesicular steatosis and features compatible with toxic parenchymal liver damage with associated bile duct damage (Figs. 1, 2, and 3).

The patient could not be weaned from the ventilator postoperatively due to the encephalopathy. Lactic acidosis resolved over a few days. Hemodialysis was discontinued after 4 days. The prothrombin time ratio increased but cholestasis persisted. Repetitive ultrasound revealed no dilated intrahepatic or extrahepatic bile ducts. The patient was weaned from the ventilator after 12 days of mechanical ventilation and could be discharged from the intensive care unit after 16 days. Bilirubin levels normalized within 14 weeks. In this period, alkaline phosphatase and gammaglutamyltransferase levels returned to the patients' baseline values (Table 1).

Meropenem was restarted 1 month later because of persisting osteomyelitis, and no increases in liver function tests were observed. The patient however died 4 months after her first ICU admission because of uncontrollable septic shock.

\section{Discussion}

DILI has been associated with the use of nearly 1,000 drugs and may mimic all forms of acute and chronic hepatobiliary disease [5, 6]. A diagnosis of DILI is expressed in terms of increasing probability, as conclusive proof is often lacking. The Council for International Organizations of Medical Sciences/Roussel Ucalf Causality Assessment Method scale [6-8] is developed to quantify the strength of the association between hepatic injury and medication as its suspected cause. The system comprises seven weighted criteria (time to onset following initial drug exposure, course of the reaction, presence of risk factors, concomitant drug exposure, exclusion of nondrug causes of liver injury, previous information regarding the known hepatotoxic potential of the drug, and response to rechallenge) in addition to the type of liver injury (hepatocellular or cholestatic/mixed). We calculated a score of 6 in our patient, which corresponds to a probable

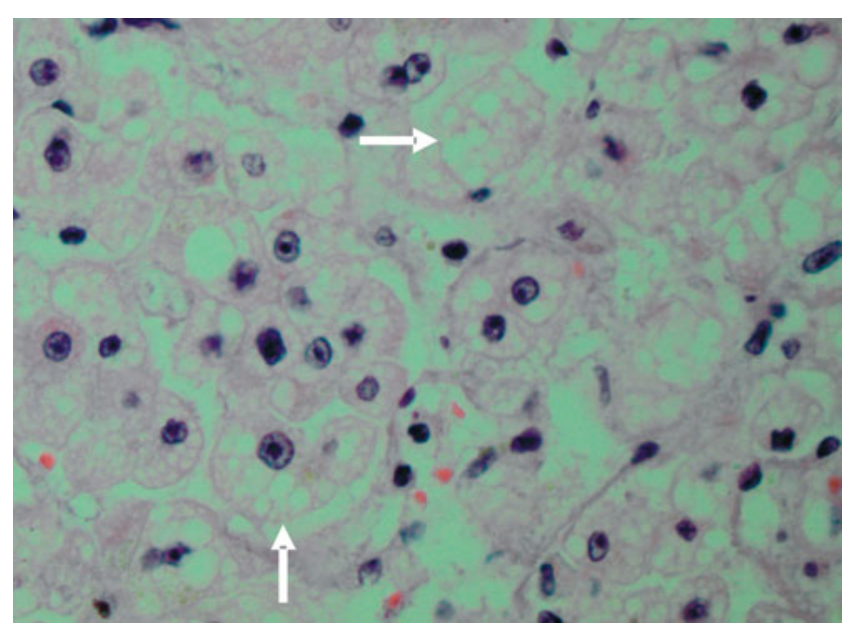

Fig. 1 The parenchyma shows diffuse microvesicular steatosis (arrows). (Original magnification $\times 400$ ) 


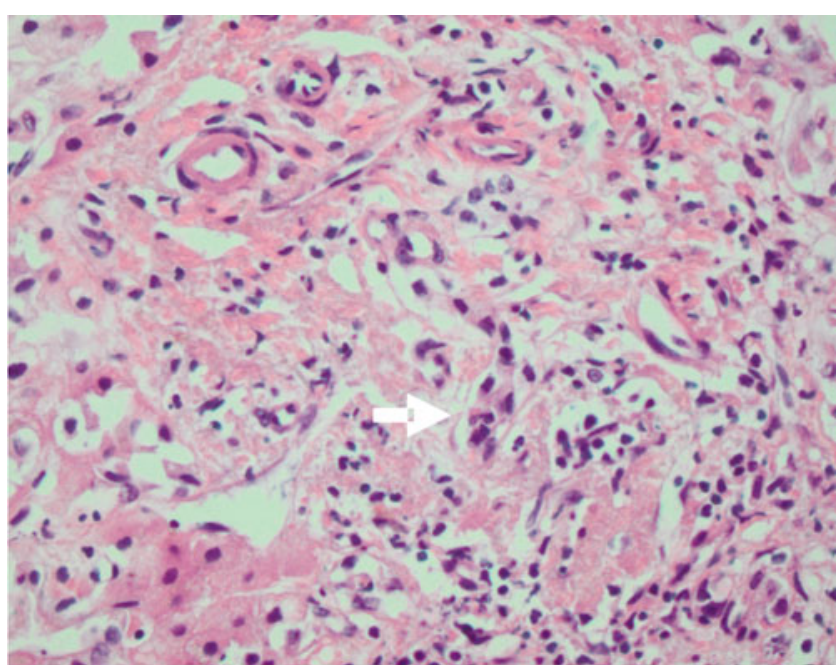

Fig. 2 Portal tract with a mildly increased mononuclear infiltrate and a bile duct with damaged, degenerated epithelium containing a lymphocyte $($ arrow). (Original magnification $\times 400$ )

association between linezolid and DILI. Elevation of liver enzymes in patients treated with linezolid has been described before [4], but severe DILI with signs of hepatic failure, as in our patient, has not yet been reported. The time course of liver dysfunction in our patient reflects that of previous case reports of patients developing linezolid-induced lactic acidosis (which was also present in our patient), both in terms of onset (after more than 6 weeks of linezolid) and of resolution following drug withdrawal and institution of supportive therapy. It is noteworthy that resolution of DILI after drug withdrawal may be delayed, in particular the cholestatic pattern which may take up to 1 year to resolve [9].

We intensively sought for alternative causes of liver injury. Screening tests for viral hepatitis, hereditary hemochromatosis, $\alpha 1$-antitrypsin deficiency, and Wilson's disease were negative, and medical imaging ruled out biliary obstruction, cardiac dysfunction, or hepatic vascular abnormality. The liver did not show ischemia on laparoscopy. Despite the presence of some anti-smooth-muscle antibodies, light microscopy of the liver biopsy revealed no autoimmune hepatitis or primary biliary cirrhosis.

In our patient, the predominant pattern of liver histology was that of diffuse microvesicular steatosis. It is identified by the presence of small, uniform lipid droplets dispersed throughout the hepatocyte, which leave the nucleus in the center of the cell. Microvesicular steatosis may, in severe cases, rapidly evolve to liver failure, coma, and death. While some hepatocytes exhibit microvesicular fat, others may exhibit macrovacuolar steatosis in the same patient. These mixed cases should be classified as microvesicular steatosis, since the latter may entail a more severe prognosis [10-12]. Various endogenous and exogenous substances impair mitochondrial ß-oxidation to cause microvesicular steatosis (for an overview, see references $[10,12])$. Our patient was not taking any of these medications, and she denied the use of any over-the-counter drugs, herbal products, or alcohol.

Microvesicular steatosis is related to severe impairment of the mitochondrial $\beta$-oxidation of fatty acids. Linezolid exerts its antimicrobial effect through inhibition of bacterial protein synthesis by binding to the $23 \mathrm{~S}$ ribosomal RNA of the $50 \mathrm{~S}$ subunit. The mitochondrial protein synthesis machinery however is in many ways similar to the prokaryotic machinery and as a result may be a collateral target for antibiotics that function by binding to the bacterial ribosome such as linezolid and tetracyclines $[2,10,13,14]$. Mitochondrial dysfunction is also the key mechanism responsible for linezolid-induced lactic acidosis [14, 15]. Finally, it should be mentioned that other antimicrobial oxazolidinones, more precisely the early derivatives investigated by DuPont in the 1980s [16], and newer potent molecules (PNU-140693 and PNU-141059) [17] are known to induce hepatic toxicity in animal models. Interestingly, in the lactic acidosis case patient of De Vrieze et al., macrovesicular and microvesicular steatoses were also seen on histological examination of liver samples. In contrast to our patient, elevation of the liver function tests or clinical symptoms associated with liver failure, with the exception of encephalopathy, were not mentioned for this case patient [14].

We considered the possibility of DILI being associated with therapy coadministered with linezolid (total parenteral nutrition and meropenem). Parenteral nutrition is a known cause of liver dysfunction, ranging from mild elevations of serum aminotransferases, alkaline phosphatase, and bilirubin to steatosis, steatohepatitis (predominantly in adults), and cholestasis (predominantly in infants). When steatosis

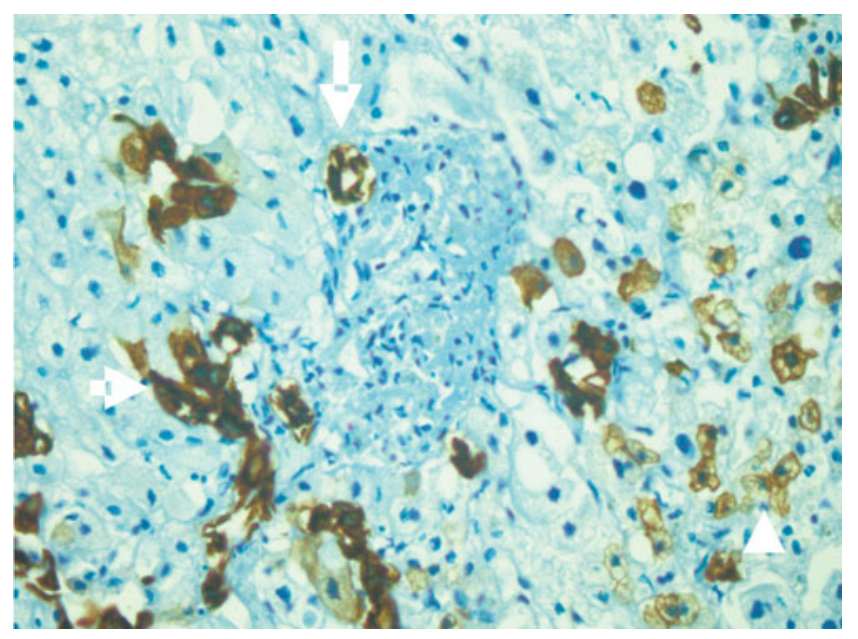

Fig. 3 The CK7 staining shows a portal tract with a damaged bile duct (long arrow), periportal ductular reaction (short arrows), and positive periportal hepatocytes, indicating cholate stasis (arrowhead). Note that the stained hepatocytes appear vacuolated due to the microvesicular steatosis. (Original magnification $\times 400$ ) 
is related to the use of parenteral nutrition, macrovesicular steatosis is the predominant finding observed on biopsy specimen [18]. Scarce articles describe both microvesicular and macrovesicular steatoses with the use of parenteral nutrition [19]. The purely microvesicular steatosis with associated bile duct damage in our patient, coupled with the fulminant course of liver dysfunction, argues against parenteral nutrition as the main etiology. The possibility remains however that total parenteral nutrition has contributed to the development of severe DILI in our patient. Elevation of liver function tests, especially transaminases, during meropenem therapy has been described in $2 \%$ to $5 \%$ of the treated patients [20]. A more thorough search of the literature showed no reports on severe hepatic failure or microvesicular steatosis. In our patient, meropenem rechallenge was not followed by recurrence of elevation of the transaminases.

The fact that our patient presented with signs of cholestasis 2 months prior to starting linezolid is a limitation in this report. As our patient already had received multiple antibiotic regimens and parenteral nutrition, the presence of an underlying preexisting liver injury is possible. However, from the time course of liver function tests, it shows that treatment with linezolid coincided with the occurrence of a new hepatotoxic event.

In conclusion, we believe that, in our patient, DILI with fulminant liver failure and lactic acidosis were probably related to prolonged use of linezolid. Clinicians should be aware of this rare but life-threatening adverse effect.

Conflict of interest The authors state that there are no potential conflicts of interest or funding.

\section{References}

1. Falagas ME, Vardakas KZ (2008) Benefit-risk assessment of linezolid for serious Gram-positive bacterial infections. Drug Saf 31:753-768

2. Bishop E, Melvani S, Howden BP, Charles PGP, Grayson ML (2006) Good clinical outcomes but high rates of adverse reactions during linezolid therapy for serious infections: a proposed protocol for monitoring therapy in complex patients. Antimicrob Agents Chemother 50:1599-1602

3. Manfredi R (2006) Update on the appropriate use of linezolid in clinical practice. Ther Clin Risk Manag 2:455-464

4. Vinh DC, Rubinstein E (2009) Linezolid: a review of safety and tolerability. J Infect 59(S1):S59-S74

5. William ML (2003) Drug-induced hepatotoxicity. N Engl J Med 349:474-485

6. Abboud G, Kaplowitz N (2007) Drug-induced liver injury. Drug Saf 30:277-294

7. Lucena MI, Camargo R, Andrade RJ et al (2001) Comparison of two clinical scales for causality assessment in hepatotoxicity. Hepatology 33:123-130

8. Garcia-Cortés M, Lucena MI, Pachkoria K et al (2008) Evaluation of Naranjo adverse drug reactions probability scale in causality assessment of drug-induced liver injury. Aliment Pharmacol Ther 27:780-789

9. Hussaini SH, Farrington EA (2007) Idiosyncratic drug-induced liver injury: an overview. Expert Opin Drug Saf 6:673-684

10. Fromenty B, Pessayre D (1997) Impaired mitochondrial function in microvesicular steatosis. J Hepatol 26:43-53

11. Fromenty B, Berson A, Pessayre D (1997) Microvesicular steatosis and steatohepatitis: role of mitochondrial dysfunction and lipid peroxidation. J Hepatol 26:13-22

12. Labbe G, Pessayre D, Fromenty B (2008) Drug-induced liver injury through mitochondrial dysfunction: mechanisms and detection during preclinical safety studies. Fundam Clin Pharmacol 22:335-353

13. McKee EE, Ferguson M, Bentley AT, Marks TA (2006) Inhibition of mammalian mitochondrial protein synthesis. Antimicrob Agents Chemother 50:2042-2049

14. De Vriese AS, Van Coster A, Smet J et al (2006) Linezolidinduced inhibition of mitochondrial protein synthesis. Clin Infect Dis 42:1111-1117

15. Garrabou G, Soriano A, Lopez S et al (2007) Reversible inhibition of mitochondrial protein synthesis during linezolid-related hyperlactatemia. Antimicrob Agents Chemother 51:962-967

16. Livermore DM (2000) Quinupristin/dalfopristin and linezolid: where, when, which and whether to use? J Antimicrob Chemother 46:347-350

17. McKee EE, Ferguson M, Bentley AT et al (2006) Inhibition of mammalian mitochondrial protein synthesis by oxazolidinones. Antimicrob Agents Chemother 50:2042-2049

18. Brunt EM (2007) Non alcoholic fatty liver disease. In: MacSween RNM, Burt AD, Portmann BC, Ferrell LD (eds) MacSween's pathology of the liver, 5th edn. Elsevier Health Sciences, Amsterdam, pp 367-398

19. Ukleya A, Romano MM (2007) Complications of parenteral nutrition. Gastroenterol Clin N Am 36:23-46

20. Linden P (2007) Safety profile of meropenem. Drug Saf 30 (8):657-668 